\title{
Skin Darkening in COVID-19 Patients: Case Series of Eight Patients in a Hemodialysis Unit.
}

\author{
Habib Ullah Rind, Nadia Iftikhar, Fazal ur Rehman, Abdul Kareem Zarkoon, Syed Mohkam, Gulalai Rehman, Umar baig, \\ Moheudin Khan, Ghulam Muhammad, Attaullah.
}

Department of Nephrology, Balochistan Institute of Nephro-Urology, Quetta, Pakistan.

\begin{abstract}
The Pandemic of COVID-19 has grasped the whole world and changed dramatically, together with our social, occupational and personal life with high mortality and morbidity with other consequences since December 2019 started from Wuhan and then declared pandemic in March. It has been observed to involve every organ of the body including skin. Various skin manifestations, but most cases reported were like urticarial rash, exanthemas, chickenpox-like vesicle, vasculitidic type, pressure sores, contact dermatitis and a single case was reported in china with skin darkening. The COVID-19 and cutaneous manifestations are recognized by physicians and dermatologist treating COVID-19. We have observed around eight cases of COVID -19 who skin darkening in our center Baluchistan Institute of Nephrology and Urology Quetta, among these included were six dialysis technicians one chronic maintenance dialysis patient and a physician. We present the representative cases and discuss the skin manifestations of COVID-19.
\end{abstract}

Key Words: COVID-19, corona virus, skin, cutaneous, darkening, hyperpigmentation, lymphopenia, hemodialysis.

\section{Corresponding Author}

Dr. Habib Ullah Rind

Department of Nephrology

Balochistan Institute of Nephro-Urology,

Quetta, Pakistan

Email: habeebaloch@gmail.com

Received: June 27, 2020. Accepted July 05, 2020.

PJKD 2020;4(3):276-279

\section{Introduction}

At the end of 2019, a novel coronavirus was identified as the cause of a cluster of pneumonia cases in Wuhan, a city in the Hubei Province of China. It rapidly spread, resulting in an epidemic throughout China, followed by an increasing number of cases in other countries throughout the world. In February 2020, the World Health Organization designated the disease COVID-19, which stands for coronavirus disease 2019.

The etiologic pathogen identified as an RNA virus belonging to the Coronavirdae is a beta coronavirus in the same subgenus as the severe acute respiratory syndrome (SARS) virus and named (Novel coronavirus SARS-Cov-2). It was isolated from Lower respiratory tract, and the resultant disease was termed as COVID-19. ${ }^{2}$ Suspicion of COVID-19 is mainly made on clinical sign/ symptoms and other laboratorial parameters. ${ }^{2}$ Dermatological manifestation of COVID-19 has different presentation reported from different part of the World, like maculopapular rash, varcilliform eruption, vasculitidic type, pressure sores, chilblain like, urticaria and contact dermatitis. ${ }^{3-6}$ In all these cases not a single case of darkening of skin was mentioned. In our literature and internet search we found one news report of a single case reported in china with skin darkening during COVID 19 infection.

We noticed changes in skin pigmentation as darkness of skin of a COVID -19 patient that resolved with a negative RT- PCR result. We suspected COVID -19 in 3 other cases based on skin color change without any febrile illness and retrospectively identified 4 other patients positive for COVID-19 where skin changes were observed in our dialysis unit. Among these included were six dialysis technicians one chronic maintenance hemodialysis patient and a physician taking care of them. We have observed all of them has developed skin darkness after average of 4 days \pm 2 days of symptoms onset or diagnosis. We present here four cases as the other patients were retrospectively analyzed and could not have their pictures for comparison.

\section{Case 1:}

The index case was a 34 years gentle man with no known comorbidity and dialysis technician by profession. He developed cough, fever >100-101 degree Fahrenheit, body aches and fatigability. His baseline labs were done with WBC count was 4320 per mm $\mathrm{mm}^{3} \mathrm{Hb} 13.9 \mathrm{~g} / \mathrm{dl}$ and platelets were normal. He was asked to do his other baseline investigations, which were all normal including X ray chest. CRP 12.3 $(<5)$ was high, d-dimers were normal and Ferritin $122 \mathrm{ng} / \mathrm{ml}$. He had RT-PCR of nasopharyngeal swab for COVID -19 done that came positive after 48 hours. He was noticed by his colleagues as having darkening of the skin by fourth day of symptoms (Figure 1: A). No previous history of skin darkening was present and he did not have any allergies or medications intake. He was recently started on multivitamins, zinc and parecetamol, for his fever. His symptoms improved by $16^{\text {th }}$ day and skin color darkness improved (Figure 1: B) only after 20 days. His RT-PCR repeated also came back negative while he was still using only zinc and multivitamin. 


\section{Case 2:}

This young 31year old dialysis technician was noted by the head technician to have skin darkness as he had fair complexion Figure 3. Based on the previous experience, he was isolated for suspicion of COVID-19 and nasopharyngeal swab for RT PCR for COVID-19 was requested.

Clinically he was asymptomatic except mild generalized weakness and early fatigability. He was treated symptomatically. Total WBC $7645 / \mathrm{mm}^{3}$, and lymphocytes: $2341 / \mathrm{mm}^{3}$, platelets: $211,000 / \mathrm{mm}^{3}$ and normal CRP. His rest of lab investigations and chest X-ray were normal.

Throughout this illness he was clinically stable and asymptomatic and he was advised to be isolated at home. He was advised to repeat his labs every 72 hour or if became symptomatic. His repeat RT-PCR for COVID-19 was negative at 2 weeks' time and his skin darkness had also improved (Figure 2).

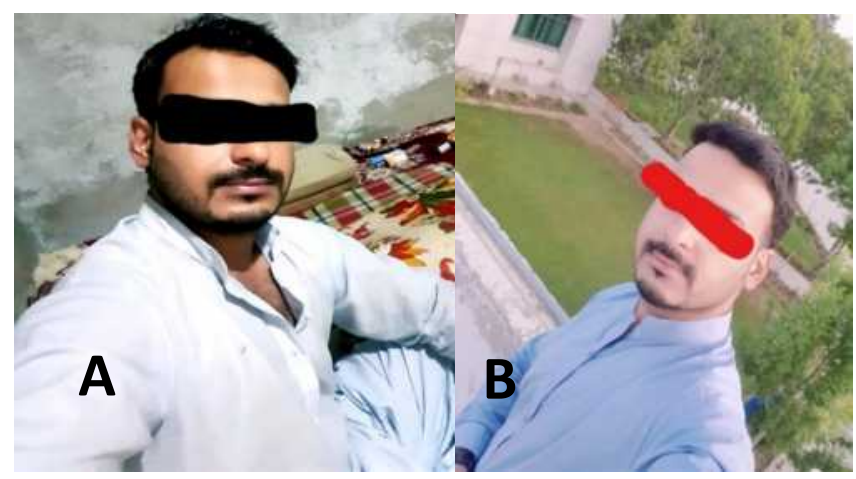

Figure 1: A) Healthy hemodialysis technician during COVID -19 illness noted with darkening of skin and B) after recovery with improvement in skin color.

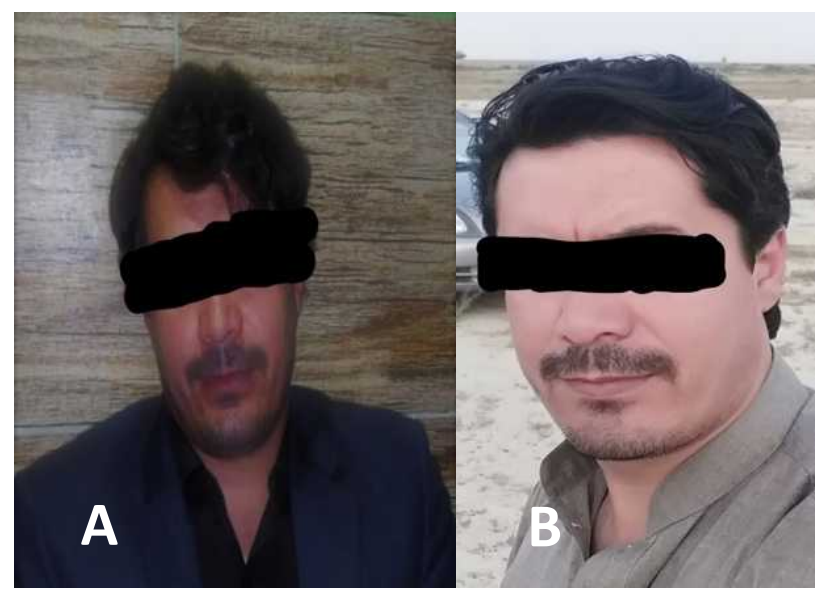

Figure 2: A) Healthy hemodialysis technician during COVID -19 illness noted with darkening of skin and B) after recovery with improvement in skin color.

\section{Case 3:}

A 45 years lady on maintenance hemodialysis in our dialysis unit was observed with recurrent hypotension and inadequate dialysis for last one week. She had been doing fairly well and did not have any symptoms of fever, aches pain etc. She was reviewed by the on-call nephrologist and admitted for evaluation of her current hypotensive issues. He also observed the skin darkness and was asked to evaluate the presence of COVID-19 based on the skin darkness and ours experience. The laboratory reports revealed leucopenia $3244 / \mathrm{mm}^{3}$ and predominantly lymphopenia, thrombocytopenia: $131000 / \mathrm{mm}$, liver function test was mildly deranged with alanine aminotransferases: 67, CRP: $122 \mathrm{mg} / \mathrm{dl}$, lactate dehydrogenase: 756u/1, D-dimer $870 \mathrm{u} / \mathrm{l}$ and X-ray chest revealed bilateral infiltrates. Patient developed dyspnea and desaturated to $<80 \%$ with $10 \mathrm{~L}$ of oxygen. She was immediately shifted to intensive care unit for inotropic support. She was prescribed broad spectrum antibiotics and inotropic support. Next six hours she did not respond to the oxygenation and inotropic support and was suspected for COVID-19 and shifted to isolation facility for ventilatory support. She was 


\section{COVID-19 and Skin Darkening}

intubated and maintained on inotropic support but unfortunately by next day she died due to unresponsive shock and ARDS. Her COVID-19 later was positive.

\section{Case 4:}

35 year old male hemodialysis technician complained of fatigability and fever. His colleague noticed his skin darkening; being a fair colored person (Figure 3) and advised him home isolation and test for COVID-19, that came back positive later. His investigations

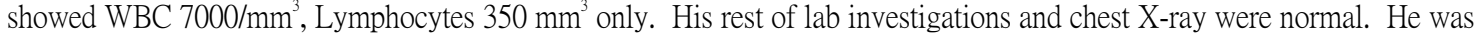
symptomatically treated and improved in the next 5 days. His repeat test came back negative 2 weeks later.

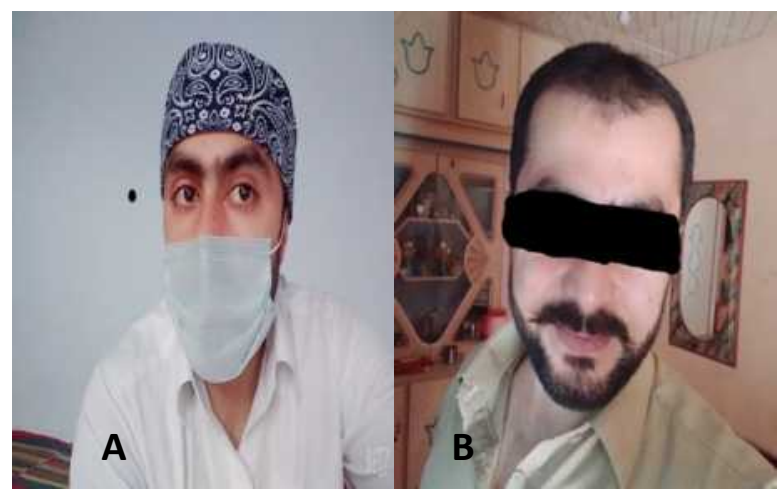

Figure 3: A) Healthy hemodialysis technician during COVID -19 illness noted with darkening of skin and B) after recovery with improvement in skin color.

The other 4 patients who were dialysis technicians and a physician were retrospectively questioned and found to have skin color changes during the episode of COVID-19 positive illness. All of them reported improvement by the time of RT-PCR for COVID-19 was negative.

\section{Discussion:}

The SARS-Cov-2 virus (COVID-19) disease has many systemic manifestations affecting almost every organ of body including dermatological manifestations. There is still little information about the evolution and mechanism of the cutaneous lesions and experience is being gained with the reports of cases from different countries. ${ }^{5}$ This is possibly the second report and the first retrospective case series of COVID-19 with this type of clinical manifestations. The time correlation of appearance and then resolution of skin darkening highly suggest COVID-19 as the possible cause and skin darkening is a new observed manifestation along with other cutaneous involvement already reported.

Skin pigmentation due to medications such as antimalarials, chemotherapeutic agents besides few others is well known and our patients were not exposed to any of these medications. ${ }^{8,9}$ Many of the endocrine disorders could cause hyperpigmentation, however our affected patients were healthy before and the hyperpigmentation was short lived and resolved with the seronegative status of COVID-19. ${ }^{10}$

In our patients the skin involvement during the course of COVID-19 may be more than a mere coincident. Another interesting observation was the skin darkening unrelated to the disease symptomatology since of the patients were suspected before the appearance of any symptoms. Since it was a diffuse change of the skin, we believe that the process involved may be systemic and possibility of hormonal changes exists as a cause. Nevertheless, it is still a speculation and needs further elaboration with hormonal assays.

Liver dysfunction during viral illness is common leading to jaundice and skin darkening, however none of our patients had significant liver dysfunction. ${ }^{11}$ Whether it is related to iron metabolism disturbances leading to high serum iron levels and skin deposition is another possibility. ${ }^{12}$

So far the reported cutaneous manifestation of COVID-19 disease are of variable nature and include urticarial rash, exanthemas, chickenpox-like vesicle vasculitic type, pressure sores, contact dermatitis, chilblains and a few more as characteristics. ${ }^{6}$ It is essential that we improve upon our knowledge of cutaneous manifestations caused by COVID-19 as a step towards early suspicion of the disease and diagnose promptly to help in decreasing its spread. Addressing these issues is necessary, yet also challenging, because there are few direct data and raises many critical issues in diagnosis. As nephrologists these subtle signs in our already multiple comorbid patients is helpful for early suspicion and isolation of patients since COVID-19 will take some time to disappear.

\section{Conclusion}




\section{COVID-19 and Skin Darkening}

In this observational case series 8 patients diagnosed with COVID-19 we noted skin darkening in the early stage of disease that resolved with the negative RT-PCR for COVID-19.

Close observation in the future for skin darkening could help in identification of suspected cases if noticed earlier, and early isolation to avoid silent spread of COVID-19.

\section{References}

1. World Health Organization. Director-General's remarks at the media briefing on 2019-nCoV on 11 February 2020. https://www.who.int/dg/speeches/detail/who-director-general-s-remarks-at-the-media-briefing-on-2019-ncov-on-11-february-2020 (Accessed on June 16, 2020).

2. Guan WJ, Ni ZY, Hu Y, Liang WH, et al. Clinical characteristics of coronavirus disease 2019 in China. New Engl J Med 2020:382:17081720

3. Sachdeva M, Gianotti R, Shah M, Lucia B, Diego Tosi, Veraldi S, et al. Cutaneous manifestations of COVID-19: Report of three cases and a review of literature J Dermatol Sci. 2020 May; 98(2): 75 - 81.

4. D. Henry, M. Ackerman, E. Sancelme, A. Finon, E. Esteve.Urticarial eruption in COVID-19 infection. J Eur Acad Dermatol Venereol 2020 34(6):e244-e245. doi: 10.1111/jdv.16472.

5. Casas, CG, Catal A, Hernandez CG, Jimenez PR, Nieto DF, Lario ARV, et al. Classification of cutaneous manifestations of COVID-19: A rapid prospective nationwide consensus study in Spain with 375 patients. Br J Dermatol 2020: 183:3-

6. Young S, Fernandez AP. Skin manifestations of COVID-19.Clev Clin J Med 2020, DOI:https://doi.org/10.3949/ccjm.87a.ccc031

7. https://www.theweek.in/news/world/2020/06/02/wuhan-doctor-whose-skin-turned-dark-from-covid-19-treatment-dies.html

8. Geddes ER, Cohen PR. Antineoplastic agent-associated serpentine supravenous hyperpigmentation: superficial venous system hyperpigmentation following intravenous chemotherapy. South Med J. 2010;103(3):231

9. Masson Regnault M, Gadaud N, Boulinguez S, Tournier E, Lamant L, Gladieff L, et al. Chemotherapy-Related Reticulate Hyperpigmentation: A Case Series and Review of the Literature. Dermatology. 2015;231(4):312.

10. Murphy-Chutorian B, Han G, Cohen SR. Dermatologic manifestations of diabetes mellitus: a review. Endo Metab Clin North Am. 2013 Dec;42(4):869-98.

11. Patel AD, Katz K, Gordon KB. Cutaneous Manifestations of Chronic Liver Disease. Clin Liver Dis - (2020): in press. https://doi.org/10.1016/j.cld.2020.04.003

12. Cavezzi A, Troiani E, Corrao S. COVID-19: hemoglobin, iron, and hypoxia beyond inflammation. A narrative review. Clinics and Pract 2020;10(1271):24-30 